\title{
Sucht als Abwehrorganisation. Perspektiven einer operationalisierten psychodynamischen Diagnostik der Sucht
} Dieter Nitzgen

\author{
Addiction as a Defensive Organisation. An Attempt at Operationalysing \\ the Psychodynamic Diagnostics of Addiction
}

\section{Zusammenfassung}

Die vorliegende Arbeit stellt die Frage nach der Möglichkeit eines psychodynamisch stringenten Suchtbegriffs. Im Mittelpunkt steht dabei der Vorschlag von R. Voigtel, Sucht psychodynamisch als „Überlassung an ein unbelebtes Objekt“ zu bestimmen. Dieser Vorschlag wird kritisch diskutiert und mithilfe der operationalisierten psychodynamischen Diagnostik (OPD) auf seine mögliche „Operationalisierbarkeit“ hin überprüft, wobei die Verwendung der OPD in der Suchtdiagnostik erläutert wird. Zusammenfassend wird die süchtige Psychodynamik abschließend als Ausdruck einer komplexen „Abwehrorganisation“ charakterisiert und nicht nur als Resultat eines einzelnen, suchtspezifischen „Mechanismus“.

\section{Schliuisselwörter}

Abwehrorganisation - Diagnostik der Sucht - operationalisierte psychodynamische Diagnostik (OPD) · Psychodynamik der Sucht

\section{Abstract}

The present paper focusses on the possibility of a psychodynamic conception of addiction. Starting from R. Voigtel's suggestion to define addiction psychodynamically as a 'surrender to an inanimate object', this assumption is being critically examined with special reference to its possible 'operationalisation'. To do so, the instrument of the 'Operationalised Psychodynamic Diagnostics' (OPD) is being employed and its use in the diagnostic process of addiction elucidated. Finally, the dynamics and structure of the addictive process are characterised as the outcome of a complex 'defensive organisation', which diagnosis is greatly facilitated by using the OPD.

\section{Key words}

Defensive organisation - diagnostics of addiction - operationalised psychodynamic diagnostics (OPD) - psychodynamics of addiction

\section{Vorbemerkung}

Anstoß für diese Arbeit war die Anwendung der operationalisierten psychodynamischen Diagnostik (OPD) [1] im Rahmen der stationären Rehabilitation Suchtkranker. Die routinemäßige Anwendung der OPD als Teil der Persönlichkeitsdiagnostik (überwiegend) alkoholabhängiger Patienten [2] hat in der Folge und im Zusammenhang mit eigenen früheren Überlegungen zur Psychodynamik der Sucht [3] die Frage nach einer möglichen „Operationalisierung“ des psychodynamischen Suchtverständnisses angeregt. Der Versuch einer solchen Operationalisierung erscheint allein deshalb sinnvoll, weil die psychoanalytischen Vorstellungen zur Genese und Dynamik der Sucht durchaus hetero- gen sind und ihre Diagnostik daher, wie Lindner [4] unlängst dargelegt hat, durch ein „Ineinander von Konflikt- und Strukturpathologie“ charakterisiert wird. Dass dieses „Ineinander“ leicht zum „Durcheinander“ bzw. zum bloßen „Nebeneinander“ zu werden droht, wird deutlich, wenn etwa ein Autor wie Rost in seiner Monographie zur Psychoanalyse des Alkoholismus [5] dessen klinische Erscheinungsformen gewissermaßen additiv ichpsychologisch, selbstpsychologisch und objektbeziehungstheoretisch konzeptualisiert, ohne sich tatsächlich um eine Vermittlung dieser Positionen zu bemühen. Insofern als nicht nur teilweise antagonistische klinische Konzepte, sondern auch durchaus widersprüchliche Behandlungskonzeptionen nebeneinander gestellt werden, ist dies nicht nur theoretisch, sondern 
vor allem auch behandlungspraktisch unbefriedigend. Von da aus stellt sich die Frage, ob und wie ein grundlegendes psychodynamisches Verständnis von Sucht formuliert werden könnte.

\section{Zum psychodynamischen Verständnis der Sucht:} Probleme und Perspektiven

Ausgangspunkt eines spezifisch „psychodynamischen“ Suchtverständnisses ist die klinisch begründete Beobachtung etwa Kernbergs [6], „daß die psychische Wirkung von Alkohol und Drogen im Einzelfall verschieden (ist), je nachdem welche intrapsychische Struktur dem Abusus jeweils zugrundeliegt“" (vgl. [7]). Das heißt, dass die psychische Wirkung von Suchtmitteln nicht ausschließlich pharmakologisch erklärbar ist, sondern zugleich auch auf einem psychologischen Wirkmechanismus basiert. Deshalb beinhaltet jeder Versuch, Sucht psychodynamisch verstehen zu wollen, die Aufgabe, diesen „Mechanismus“ psychodynamisch schlüssig zu explizieren. Dies stößt in mehrfacher Hinsicht auf Schwierigkeiten und Probleme. Zunächst aufgrund der Komplexität des Gegenstandes, insofern es sich hier immer um ein komplexes Ineinander von somatischen, sozialen und psychologischen Prozessen handelt [8] und die Sucht als Phänomen den Geltungsbereich bestehender akademischer Disziplinen überschreitet. Dessen eingedenk muss auch ein psychodynamisches Verständnis von Abhängigkeit und Sucht davon ausgehen, dass ,im besten Fall ... die Befunde des einen Systembereichs Eingangs- oder Ausgangsgrößen für den anderen (sind)“, wie R. Krause [9] in seiner allgemeinen psychoanalytischen Krankheitslehre festgestellt hat. So wäre es Krause [10] zufolge etwa „naiv“ anzunehmen, dass die „pharmakologische Wirkung“ eines Suchtmittels „von den Phantasien, zu deren Handhabung sie eingesetzt wird, unabhängig sei“ (vgl. [11]). Daraus folgt, wie Heigel-Ewers et al. [12] betont haben, dass psychodynamische Annahmen grundsätzlich als „notwendige, wenngleich nicht als hinreichende Bedingungen für die Entwicklung einer Abhängigkeit anzusehen sind“. Definitorische Probleme ergeben sich im Weiteren aus der „Uneinheitlichkeit“ der Suchtmittel, ihrem stofflichen und nichtstofflichen Charakter. Bekanntlich gibt es neben den „klassischen“ stoffgebundenen Süchten wie Alkohol-, Medikamenten- und Drogenabhängigkeit auch „stoffungebundene“ Süchte wie etwa die Spielsucht. Als zusätzlich komplizierend erweist sich, dass zentrale Aspekte des Suchtgeschehens wie etwa das drängende Verlangen zu konsumieren (craving) bzw. der zwanghafte Charakter süchtigen Handelns klinisch auch bei anderen, nicht unmittelbar mit den Suchterkrankungen assoziierten Störungsbildern und Syndromen feststellbar sind, etwa bei den sexuellen Perversionen und den Zwangsstörungen [13]. Eben weil die Sucht nach einem Wort A. Freuds [14] ein „ein hoch zusammengesetztes, symptomatisches Gebilde“ ist, stellt sich diagnostisch umso dringlicher die Frage nach ihrer Spezifität. Lässt sich überhaupt ein Kriterium angeben, das es erlauben würde, Sucht psychodynamisch so zu definieren, dass sie von anderen Störungsbildern abgrenzbar wird? Mit Blick darauf hat R. Voigtel unlängst in seinen Ausführungen zur psychoanalytischen Diagnostik der Sucht [15] dargelegt, dass es diagnostisch wesentlich darum geht, Sucht so zu definieren, „daß sie sich auch nach den Rändern hin mit gebotener wissenschaftlicher Schärfe abgrenzt“. Angesichts dieser Problematik konstatiert dieser Autor zunächst eine gewisse „Beliebig- keit bei der Bestimmung dessen, was eigentlich Sucht sei“ in der vorgängigen psychiatrischen und psychoanalytischen Literatur. Einerseits würde der Suchtbegriff hier so erweitert, dass praktisch „jede menschliche Leidenschaft süchtig entarten könne“ [16], wodurch er letztlich unspezifisch wird. Andererseits aber würde er nach Maßgabe von äußerlich-empirischen Merkmalen wie etwa Menge und Art des Konsums bestimmt, die aus psychodynamischer Sicht als Kriterien für eine Grenzbestimmung unbefriedigend bleiben müssen. „Wo“, so fragt Voigtel [17], ,ist die Grenze zwischen einer psychisch stabilisierenden Gewohnheit (die täglichen zwei Gläser Wein) und einem autodestruktiven Zwang (drei Gläser Wein)?" Fängt die Sucht bei zwei oder zwanzig Zigaretten pro Tag an? Entsprechenden Versuchen, süchtiges Handeln rein quantitativ zu definieren, hält er entgegen, dass „Dauer und Gewohnheit eines Tuns ... es noch nicht zur Sucht machen; ebensowenig Zwanghaftigkeit und Entstehung von Angst bei Entzug dieses Tuns ....". Aber auch die Orientierung an Merkmalen wie der Menge des Konsums bestimmter Drogen oder der somatischen oder sozialen Schädlichkeit eines Tuns für einen Menschen ist seiner Ansicht nach für die Abgrenzung einer Krankheitseinheit „Sucht“ überflüssig“, weshalb er provozierenderweise hinzufügt:

„Wenn wir uns nun mit der empirischen Tatsache auseinandersetzen, daß es eine Reihe von Menschen gibt, die von Glücksspiel genauso abhängig sind wie von einer Droge, einschließlich des Kater-Gefühls und der Entzugserscheinungen, mit den gleichen Glückshoffnungen und der gleichen willenlosen Hingabe und dem gleichen faktischen Ruin, dann müssen wir uns zunächst von dem Gedanken verabschieden, daß die faktische Inkorporation eines Objekts ein Suchtkriterium sei. Wir müssen davon ausgehen, daß die toxische oder chemische Wirkung inkorporierter Stoffe für den Suchtmechanismus nicht wesentlich ist ..."

Wenn es weder die „faktische Inkorporation“ noch die „chemischen“ bzw. „toxischen“ Wirkungen des Suchtmittels sind, die dessen psychische Wirkung bestimmen, worin besteht dann das psychodynamisch Wesentliche des „Suchtmechanismus“? Grundsätzlich klärend war hier die Einsicht Simmels [18], der in seinen psychoanalytischen Ausführungen zur Alkoholsucht schon 1948 feststellte:

„Daß das Bedürfnis, alkoholische Getränke zu sich zu nehmen, dem einen Mittel als Mittel zur Realitätsflucht dient, während der andere es zur Realitätsbewältigung verwendet, beweist, daß das der ausschlaggebende Faktor, nicht die biochemische Wirkung des Alkohols ist, sondern die psychologische Wirkung, die das Ich daraus bezieht.“

Voigtel greift Simmels Grundannahme vom Vorrang der psychologischen Funktion des Suchtmittels in der Suchtdynamik ein halbes Jahrhundert später wieder auf. Für ihn besteht diese psychologische Funktion wesentlich in der beruhigenden Eigenschaft des Suchtmittels infolge seines „dinglichen“ Charakters und seiner deshalb zuverlässigen „Verfügbarkeit“. „Süchtige“, so Voigtel [19], ,ertragen die Enttäuschungen des Lebendigen nicht." Zur Erläuterung zitiert er die Aussage einer Patientin, die ihm während der Behandlung sagte: „Ich möchte mich von keinem (Menschen) so abhängig machen - höchstens von Gegenstän- 
den“. ${ }^{1}$ Der süchtige „Ausweg“ angesichts von Enttäuschungen durch eine fehlbare und allzu fehlbare menschliche Mitwelt besteht demzufolge darin, „sich unbelebten Objekten zu überlassen, da diese nicht nur ... zuverlässig sind, sondern darüber hinaus weder kränken noch unerfüllbare Forderungen und damit wiederum kränkende Forderungen stellen können ...“ Es ist also diese „Überlassung an ein unbelebtes Objekt“, die für den psychodynamischen Modus des Suchtgeschehens spezifisch ist. Sie vollzieht sich im Zeichen der projektiven Zuschreibung menschlicher Qualitäten, die das Suchtmittel in der Folge zum Träger dieser Qualitäten erhebt, von denen das Selbst nun die Linderung seiner Ohnmachts- und Verlassenheitsgefühle bzw. Glück und Wohlbefinden erwartet und sich ihm in dieser Erwartung passiv ausliefert. Struktureller Hintergrund dafür ist eine tief greifende „Passivität des Selbst“, die für Voigtel [21] zum „Wesen“ der Sucht gehört. Wie er anhand einer gründlichen Revision der relevanten Literatur aufzeigt, korreliert diese „Passivität“ mit einer mehr oder weniger generellen, strukturellen Beeinträchtigung des süchtigen Subjekts, die je nach den leitenden theoretischen Vorstellungen der Autoren als „basale Verstimmung“ bzw. Ich-Schwäche bzw. narzisstisches Defizit bzw. autodestruktive Tendenz beschrieben wird.

„Für die schweren Fälle von Sucht haben wir“, so Voigtel, „einen Mangel an Selbstwertgefühl konstatiert, eine fehlende Ich-Fähigkeit, Affekte differenziert zu erleben und zu kommunizieren, diffuse Angst, ein Ohnmachts- und Schuldgefühl, schließlich Haß auf die eigene Unfähigkeit und Schlechtigkeit und, dazu passend, ein negatives Selbstbild.“

Im Hinblick darauf, dass sich diese strukturellen Beeinträchtigungen auch im Rahmen anderer Störungsbilder wiederfinden lassen, betont Voigtel aber konsequent, dass das entscheidende Kriterium, welches Sucht von anderen narzisstischen Störungen unterscheidet, „auch wenn sie ihr äußerlich sehr ähnlich sehen ...“, der Mechanismus der „Hingabe an ein unbelebtes Objekt“ ist. Daher erscheint die Sucht für ihn eben nicht (nur) als Ausdruck eines persönlichkeitsstrukturellen Defizits (wie etwa für Kohut), sondern „im klassischen Freud'schen Sinne als Symptom einer Kompromißbildung“. Sucht ist „nicht nur das Ausfüllen eines narzißtischen Defizits oder eine gradlinige, vom Über-Ich geleitete Selbstzerstörung, sondern einerseits ein agierter Abhängigkeitswunsch (Trieb) und andererseits dessen Abwehr durch Verschiebung auf ein unbelebtes Objekt“. Insofern dieser (Abwehr-)Vorgang der Verdrängung unterliegt, imponiert süchtiges Verhalten in der Folge als „bewußtloser, zwanghafter Ablauf“. Damit gelingt es Voigtel, die Psychodynamik der Sucht stringent zu formulieren. Seine Argumentation ist dabei dem von Heigel-Evers, SchulzeDierbach u. Standke [22] zuvor unternommenen Versuch vergleichbar, „suchtspezifische Objektbeziehungsmuster“ zu identifizieren, erscheint aber psychodynamisch insofern stringenter, als sie nicht nur die „Passivität“ des Selbst gegenüber dem Objekt thematisiert, sondern darüber hinaus den spezifischen „Modus“ (Mechanismus) der Suchtdynamik benennt. Dieser besteht eben nicht (nur) darin, dass der Süchtige sich dem realen und/oder ima-

\footnotetext{
${ }^{1}$ Das damit verbundene Paradoxon hat S. Geismar-Wieviorka [20] in ihrer Rekonstruktion des süchtigen Denkens formuliert, wenn sie einen Wunsch nach „Superunabhängigkeit“ bei Süchtigen feststellt: „Sie glauben an die absolute Unabhängigkeit, und da sie diese nicht erreichen können, ziehen sie eine völlig abhängige Lebensweise vor.“
}

ginären menschlichen Anderen der unbewussten Phantasie passiv „überlässt“, wie diese annehmen, sondern sich dem Suchtmittel als einem „Ding“ ausliefert. Dem entspricht ätiologisch die von Heigel-Evers, Standke und Wienen [23] geäußerte Vermutung, dass in der Genese der Suchterkrankung „möglicherweise schon sehr früh in der kindlichen Entwicklung zur Befriedigung von Triebwünschen Angebote gemacht wurden, die unpersönliche Mittel zur Beseitigung von Unlustgefühlen beinhalteten“. Somit erscheint Sucht psychodynamisch als Abwehr basaler Beziehungswünsche.

Die Spezifität des süichtigen Modus im Verhältnis zu anderen Störungsbildern

Der Vorschlag, die suchtspezifische Dynamik als „Überlassung an ein unbelebtes Objekt“ zu definieren, liefert neben einem brauchbaren diagnostischen Kriterium zur kategorialen Bestimmung der Sucht auch ein dimensionales Kriterium² ${ }^{2}$, d. h. einen Hinweis auf ihren möglichen Schweregrad. Wenn, wie Voigtel [25] annimmt, „der psychische Zweck der Ausschaltung des Selbst und das Mittel der Überlassung an ein unbelebtes Objekt" die Kriterien dafür sind, ob es sich bei einer bestimmten Verhaltensweise um eine Sucht handelt oder nicht, dann lässt sich Sucht psychodynamisch von anderen Störungsbildern ebenso abgrenzen wie auch das Spektrum der Süchte differenzieren. Erfüllt werden diese Kriterien natürlich von den klassischen, stoffgebundenen Süchten wie Alkohol-, Medikamenten- und Drogensucht, aber auch von der Spielsucht, sofern hier Glücksspiele bzw. Spielen um Geld gemeint sind, d.h., dass das Subjekt sich dabei passiv „Zufallsmechanismen“ ausliefert bzw. einem hohen finanziellen Risiko aussetzt. Nicht erfüllt werden diese Kriterien, je mehr bei Spielaktivitäten „Geschicklichkeit und Können“, also Ichfähigkeiten, ins Spiel kommen und desto weniger also eine passive Auslieferung und ein „Abtöten des Selbst“ [26] stattfinden.

„Man könnte“, so Voigtel, „hier eine Reihe aufstellen, angefangen bei Spielen mit hohem Zufallsfaktor und geringer Geschicklichkeitsanforderung an den „one-arm-bandits“, dem Roulette und dem Würfeln, bis hin zu Spielen mit umgekehrten Faktoren, beispielsweise Billard oder Skat, wobei Spiele wie Poker oder normale Automatenspiele dazwischen liegen“.

Insgesamt gilt für ihn, „dass alle mit Motivation, Aktivität und manchmal mit Anstrengung des Selbst verbundenen Abhängigkeiten ..., auch wenn sie der Abwehr von psychischen oder sozialen Konflikten dienen, keine Sucht sind“. Das heißt, dass zwanghaftes Arbeiten, intensives Bodybuilding, Computerspiele bzw. exzessives Lesen und/oder Tagträumen nicht die Kriterien der Sucht erfüllt. Erfüllt werden diese Kriterien seiner Ansicht nach auch nicht von zwei anderen Störungsbildern, die üblicherweise mit der Sucht assoziiert werden, nämlich den Essstörungen. Von der Anorexie nicht, insofern hier die Selbstdisziplin des Subjekts erhalten bleibt und somit das Kriterium der Passivität verfehlt wird, und von der Bulimie nicht, insofern dabei eine „Überlassung“ an das die mütterliche Versorgung repräsentierende

\footnotetext{
2 Zu den Begriffen „kategorial“ und „dimensional“ vgl. Kernberg [24], vor allem S. 57 ff. und 61.
} 
Objekt, das Essen, „nur für Minuten“ stattfindet. Weiterhin ermöglichen die genannten Kriterien von „Passivität“ und „Überlassung“ auch eine differenzierte Betrachtung des bereits von Sachs [27] konstatierten Zusammenhangs von Sucht und Perversion. Auf diesen Zusammenhang rekurriert auch Voigtel [28], wenn er das unbelebte Suchtobjekt als quasi „Fetisch“ bezeichnet, dessen fetischistischer Charakter sich aus der ihm zugeschrieben „Anziehungskraft und Macht" ergibt. Geht man mit Cooper [29] von einer "narzisstischen Basis der perversen Entwicklung aus“, wobei deren „Kerntrauma“ im „Erleben einer schreckerregenden Passivität gegenüber der präödipalen Mutter besteht, die als gefährlich, böse, übelwollend und allmächtig gesehen wird“, dann kann eine Dynamik immer dann als „pervers“ angesehen werden, „wenn eine Handlung oder Phantasie von der Verleugnung unbewußter Passivität beherrscht wird, und zwar mittels der dreifachen Phantasien von der Verdinglichung des Objekts, der Verdinglichung des Selbst und der Bewerkstelligung masochistischer Lust“. Ausgehend von diesen drei „Grundphantasien“ des perversen Subjekts, die Cooper als den „Kern“ jeder Perversion ansieht, lassen sich die prinzipiellen Unterschiede und Gemeinsamkeiten von Sucht und Perversion genauer bestimmen. Während es in der perversen Entwicklung primär zu einer fantasierten Verdinglichung des Anderen und des Selbst kommt, also seiner imaginären „Dehumanisierung“, wie Cooper sagt, erfährt diese in der Sucht eine Radikalisierung, insofern das Subjekt sich hier nicht einem „dehumanisierten", realiter aber immer noch menschlichen Anderen überlässt, sondern sich in der Tat einem „Ding“ ausliefert, dessen menschliche Attribute nur noch imaginär sind. Für die sexuellen Perversionen trifft demzufolge zu, was Voigtel [30] mit Blick auf die Bulimie feststellt: Sie erfüllen nicht die Kriterien der Sucht im engeren Sinne, wohl aber die einer „narzißtischen Störung mit süchtigen Anteilen“. Dass dem Suchtmittel der Charakter eines fetischisierten Objekts zukommen soll, entspricht im Übrigen auch der Auffassung J. McDougalls [31], die im Anschluss an Winnicott [32] von Suchtmitteln als „pathologischen Übergangsobjekten“ spricht, die das Ergebnis gescheiterter positiver Internalisierungsprozesse sind. Dementsprechend korrespondiert für sie [33] auch die „Wahl“ eines Suchtmittels mit „... genau dem jeweiligen Entwicklungsstadium eines Subjekts, in dessen Verlauf eine Integration hilfreicher und gutartigerer Introjekte gescheitert ist". ${ }^{3}$ Wenn also die Entwicklung von Sucht und Perversion sich gleichermaßen auf das Scheitern entwicklungsfördernder Internalisierungen zurückführen lässt, imponiert die Sucht im Gegensatz zur Perversion jedoch als Fixierung auf ein „unbelebtes Objekt“ anstelle eines süchtig benötigten menschlichen Anderen. Voigtel [35] spricht demzufolge zu Recht vom Suchtmittel als einem umfassenden „Beziehungs-Fetisch“.

Was die Ausprägung einer Sucht, d. h. ihren möglichen „Schweregrad“", angeht, so wird dieser nach Maßgabe von der Disposition des Subjekts bestimmt, sich dem Suchtmittel passiv zu „überlassen“.

„In einem umfassenden Sinne“, schreibt Voigtel, „zeigt sich die Machtübernahme des Objekts darin, daß bei schwerer Sucht das Leben, einschließlich der Gedanken, um es herum organisiert ist."

\footnotetext{
${ }^{3}$ Dem entspricht auch die These Khantzians [34] von der „Wahl“ des Suchtmittels als Form einer auch pharmakologisch sinnvollen „Selbstmedikation“ (1985).
}

Das heißt, dass der Suchtverlauf psychodynamisch in dem Maße als „schwerer“ anzusehen ist, in dem die sozialen, privaten und beruflichen Vollzüge des süchtigen Subjekts zunehmend in den Hintergrund treten und stattdessen das unbelebte Suchtobjekt seine innere und äußere Welt progressiv in Besitz nimmt; zunächst punktuell im sog. „Kontrollverlust“ und in letzter Konsequenz total im Akt seiner (Selbst-)Zerstörung im und durch den Rausch. Insofern erscheint auch der Suizid, ob intendiert wie im „goldenen Schuss“ oder billigend in Kauf genommen wie etwa bei exzessiver Alkoholsucht, als durchaus logische und letzte Konsequenz des suchtspezifischen Modus der „Überlassung“ des Subjekts an ein unbelebtes Objekt (dem es sich damit i. S. einer „Identifikation mit dem Aggressor“ anverwandelt und somit selbst „unbelebt“ wird).

\section{Zur Operationalisierung des psychodynamischen Suchtbegriffs}

Was die süchtige Psychodynamik von der anderer narzisstischer Störungen unterscheidet, ist der Modus der „Hingabe an ein unbelebtes Objekt“. Dabei imponiert der suchtspezifische Modus als „Kompromißbildung“ [36], d. h. einerseits als „agierter Abhängigkeitswunsch“ und andererseits als dessen „Abwehr durch Verschiebung“. Wenn es Voigtel damit auch gelingt, die spezifisch süchtige Psychodynamik hinreichend zu bestimmen, so erweist sich seine Definition bei genauerer Betrachtung allerdings als unscharf, insofern dabei konfliktbezogene und strukturelle Gesichtspunkte unausgesprochen konfluieren. Denn zum einen wird das Suchtgeschehen konfliktdynamisch in Begriffen von „Trieb“ und „Abwehr“ konzeptualisiert; andererseits aber als strukturspezifisch durch das Merkmal der „Passivität“ bestimmt ausgewiesen. Wenn Voigtel bezüglich der Bereitschaft, sich dem Suchtmittel passiv zu überlassen, auf Begriffe wie „basale Verstimmung“ bzw. „Ich-Schwäche“ bzw. „narzisstisches Defizit“ rekurriert, wird deutlich, dass er die „Passivität“ des Süchtigen eben nicht nur konfliktdynamisch, sondern auch als Merkmal der Persönlichkeitsstruktur versteht. Damit bleibt auch seinem Suchtverständnis das von Lindner [37] festgestellte „Ineinander von Konflikt- und Strukturpathologie" in der Suchtdiagnostik implizit, ohne explizit vermittelt zu werden. Das gilt im Übrigen auch für die nosologische Klassifizierung der Sucht als Form der „narzisstischen Störung“ [38], insofern bei deren Beschreibung wiederum konflikt- und strukturpathologische Merkmale begrifflich „vermischt“ werden. Ungeachtet dieser begrifflichen Unschärfe erscheinen Voigtels Überlegungen aber als Ausgangspunkt für eine weitergehende konzeptionelle Klärung geeignet. Dazu soll im Folgenden das Instrument der operationalisierten psychodynamischen Diagnostik (OPD), ein multiaxiales Klassifikationssystem zur Erfassung psychischen Geschehens [39], herangezogen werden. Nach Auffassung der OPD-Arbeitsgruppe können psychische Phänomene psychodynamisch grundsätzlich anhand von vier Achsen klassifiziert werden (Achse V: Psychische und psychosomatische Störungen sind als Anschlussachse zur psychiatrischen ICD/DSM-Diagnostik konzipiert und spielen daher im vorliegenden Zusammenhang keine Rolle): als Krankheitserleben (Achse I), als Beziehungsgestaltung (Achse II), als Konfliktdynamik (Achse III) und als (Persönlichkeits-)Struktur (Achse IV), wobei die Besonderheit des diagnostischen Ansatzes in der „Operationalisierung“ dieser Achsen, d. h. 
ihren definitorischen Festlegungen, besteht. So steht es dem Untersucher, der mit diesem Instrument arbeitet, eben nicht mehr frei, sein eigenes Vorverständnis etwa von psychischem „Konflikt“ und „Struktur“ zu verwenden, sondern er wird diesbezüglich auf deren Operationalisierung im Manual verwiesen. Mit Bezug auf Voigtels Verständnis der Sucht als „Kompromißbildung“ vor dem Hintergrund einer tief greifenden, strukturellen „Passivität“ des Subjekts lassen sich diese beiden Dimensionen im Rahmen der OPD anhand der Achsen III (Konflikt) und IV (Struktur) erfassen. Dabei bereitet die Klassifikation der strukturellen „Passivität“ konzeptuell keine Schwierigkeit, insofern deren Merkmale sich anhand der Items der Strukturachse unmittelbar raten lassen (sollten). Das gilt so nicht für den suchtspezifischen Modus der „Überlassung an ein unbelebtes Objekt“, da dieser Modus in seiner Spezifität von der Konfliktachse nicht abgebildet wird. Wenn die Autoren der OPD das Konstrukt des psychischen „Konflikts“ i.S. des Vorhandenseins „innerer unbewusster zeitüberdauernder Konflikte“ definieren, dann sollen anhand der Konfliktachse basale intrapsychische Konfliktmuster abgebildet werden, nicht aber die Spezifität der Suchtdynamik. Dass ihre Anwendung im Rahmen der Suchtdiagnostik dennoch sinnvoll ist, ergibt sich grundsätzlich bereits aus dem konfliktdynamischen Verständnis des Suchtgeschehens als unbewusstem Abhängigkeitswunsch, der qua „Verschiebung“ auf ein unbelebtes Objekt abgewehrt und somit „agiert“ wird. Spezifisch für die Sucht ist dabei nicht der ihr zugrunde liegende Abhängigkeitswunsch, sondern seine Abwehr durch die Verschiebung auf das Suchtmittel. Insofern also nicht der Wunsch selbst, sondern seine abwehrbedingte Verarbeitung suchtspezifisch ist, erscheint auch die Annahme eines suchtspezifischen Konflikts sui generis nicht plausibel. Suchtspezifisch ist vielmehr der Modus der Verarbeitung dieses Abhängigkeitswunsches. ${ }^{4}$ Damit ist die Anwendung der Konfliktachse in der Suchtdiagnostik nicht nur grundsätzlich sinnvoll, sondern erlaubt darüber hinaus auch, den in der Sucht agierten Abhängigkeitswunsch, den Voigtel selbst nicht weiter differenziert, anhand der in der OPD operationalisierten Konfliktmuster zu spezifizieren. Indem die Beziehung des Süchtigen zum Suchtmittel ausdrücklich zum Gegenstand der diagnostischen Aufmerksamkeit gemacht wird, kann gefragt werden, welcher Wunsch denn mit der Einnahme von Suchtmitteln abgewehrt werden soll: ein Wunsch nach symbiotischer Verschmelzung, nach materieller und/oder psychischer Versorgung, nach Selbstwertregulation, Unterwerfung und/oder Schuldentlastung? Entsprechend der besonderen Natur und Intention dieses Wunsches kann die der Sucht zugrunde liegende Konfliktdynamik dann genauer als Abhängigkeits-/Autonomiekonflikt i.e.S. oder als Versorgungs-/Autarkiekonflikt, Selbstwertkonflikt, Unterwerfungs-/Kontrollkonflikt bzw. Über-Ich-/Schuldkonflikt identifi-

\footnotetext{
${ }^{4}$ Diese Definition könnte Anlass zu Missverständnissen geben, insofern die OPD [40] den Begriff „Modus der Konfliktverarbeitung“ i.S. eines habituell vorherrschenden, passiven und/oder aktiven „Modus“ der Konfliktverarbeitung definiert, also als „eine grundsätzliche Bipolarität zwischen Passivität und Aktivität, zwischen Selbst- und Objektbezogenheit“ (S. 125). Dieser bipolare Modus der Konfliktverarbeitung ist weder theoretisch noch empirisch „suchtspezifisch“; er liegt auf einer anderen theoretischen „Ebene“. Empirisch ergab sich beispielsweise in unserer Untersuchung [41] alkoholabhängiger Patienten keine eindeutige Prävalenz einer vorwiegend aktiven und/oder passiven Konfliktverarbeitung (mit Ausnahme des Versorgungs-/Autarkiekonflikts, bei dem die passive Verarbeitung eindeutig überwog; vgl. S. 243).
}

ziert werden. Davon ausgehend ergeben sich zugleich auch Hinweise auf die Globalität der Suchtdynamik, ihre „dimensionale“ Ausprägung: vom Wunsch nach globaler „Verschmelzung“, d. h. völliger „Überlassung“ des Selbst an das Suchtobjekt, über seine weniger umfassende „Versorgung“ bis hin zur noch punktuelleren „Unterwerfung“ unter das Suchtmittel (etwa im Sinne der Verleugnung der Urheberschaft eigenen Handelns).

Wie bereits angedeutet, bereitet die Erfassung der strukturellen „Passivität“ des süchtigen Ichs anhand der Strukturachse (Achse IV) der OPD prinzipiell keine Schwierigkeiten. Von den Autoren der OPD [42] wird das Konstrukt der psychischen „Struktur“ als „eine für den Einzelnen typische Disposition des Erlebens und Verhaltens“ in den Bereichen Selbstwahrnehmung, Objektwahrnehmung, Selbststeuerung, Abwehr, Kommunikation und Binding definiert, deren Funktionsniveau jeweils „gut“, „mäßig“, „gering“ oder „desintegriert“ sein kann. Mit Bezug darauf kann die Persönlichkeitsstruktur des Süchtigen über die undifferenzierte Zuschreibung einer vermeintlichen „Passivität“ hinaus im Einzelnen als strukturell verschieden integriert klassifiziert werden. Insofern sich diesen Integrations- bzw. Funktionsniveaus der Persönlichkeit psychopathologisch mit Kernberg [43] auch verschiedene „Ebenen“ der Charakterpathologie zuordnen lassen, kann auch die süchtige „Passivität“ dann strukturspezifisch als Ausdruck einer neurotischen Borderline bzw. psychotischen Persönlichkeitsorganisation ausgewiesen werden. Damit eröffnet die OPD als Klassifikationssystem die Möglichkeit, den suchtspezifischen Modus der „Überlassung an ein unbelebtes Objekt“ - als hinreichendes Kriterium der Suchtdiagnostik strukturell zureichend zu differenzieren. Dass dieser Modus strukturell auf verschiedenen Integrationsniveaus (von gut integriert bis desintegriert) bzw. „Ebenen“ der Charakterpathologie wirksam werden kann, erlaubt es, an der „Einheit“ des Suchtgeschehens, d.h. seiner psychodynamischen Spezifität gegenüber anderen Störungsbildern, festzuhalten, ohne dabei die Verschiedenheit seiner Erscheinungsformen und Schweregrade aus dem Blick zu verlieren. Sucht wird auf diese Weise mehrdimensional: als Resultante unterschiedlicher, unbewusster Konfliktkonstellationen und verschiedener struktureller Integrationsniveaus der Persönlichkeit klassifizierbar.

Was die Anwendung der OPD im Rahmen der Suchtdiagnostik betrifft, erscheint danach zunächst nicht ihre störungsspezifische Adaption vordringlich zu sein (etwa durch die Kreierung einer „suchtspezifischen“ Achse), sondern ihre gezielte „Verwendung“ im Hinblick auf die der Sucht zugrunde liegenden Konflikt- und Strukturdimensionen, wie es hier (unter bewusster Ausklammerung der Beziehungsachse $)^{5}$ geschehen ist. Daraus ergeben sich im Folgenden auch weiterführende Forschungsperspektiven. So kommt etwa Reymann [44] in seinem jüngst erschienenen Beitrag zur einer „evidenzbasierten“ psychodynamischen Therapie Alkoholabhängiger anhand seiner methodisch aufwändigen Untersuchung von 23 stationär behandelten alkoholabhängigen Patienten mit der OPD [45] zu dem Ergebnis, dass diese Patienten hinsichtlich ihres Strukturniveaus vornehmlich auf einem mäßigen (Score 1) bzw. einem geringen (Score 2)

\footnotetext{
${ }^{5}$ Insofern diese für die vorliegenden Fragestellungen nicht in dem Maße relevant ist wie die Konflikt- und Strukturachsen.
} 
Integrationsniveau angesiedelt sind, weshalb auch bei Alkoholabhängigen grundsätzlich „die Voraussetzungen für eine psychodynamische Therapie gegeben sind“. Das entspricht den empirischen Befunden unserer eigenen Untersuchung von 171 alkoholabhängigen Patienten einer Rehabilitationsklinik [46] mit einer Differenz der Standardabweichung von nur 0,4 zwischen Reymann $(0,44)$ und uns $(0,48)$. Eine weitere Übereinstimmung ergibt sich auch im Hinblick auf die empirische Korrelation zwischen Konflikt- und Strukturdimension, wie sie unlängst allgemein von Grande et al. [47] untersucht wurde. Dabei zeigten sich bei Grande et al. wie auch in unserer Arbeit [48] empirisch nachweisbare Zusammenhänge zwischen verschiedenen Konflikten und strukturellen Integrationsniveaus (etwa zwischen dem Vorliegen eines Abhängigkeits-/Autonomiekonflikts und einem tendenziell geringen Strukturniveau) wie auch signifikante $\mathrm{Zu}$ sammenhänge zwischen bestimmten Konflikten und Störungsbildern. So gingen auch bei den alkoholabhängigen Patienten neurotische Störungsbilder „verstärkt einher mit VersorgungsAutarkie sowie Unterwerfungs-Kontrollkonflikten, während Persönlichkeitsstörungen eher mit Abhängigkeits-Autonomie und Selbstwertkonflikten zusammenhingen“. Auf diese Weise wird es möglich, die durchaus heterogene Population Suchtkranker diagnostisch weiter zu differenzieren und so klinische „Subtypologien von Alkoholabhängigen" zu identifizieren, wie dies von der empirischen Forschung zur Wirksamkeit einer psychodynamisch orientierten Suchttherapie [49] heute verstärkt gefordert wird.

\section{Schlussfolgerungen}

Geht man mit Voigtel davon aus, dass der für die Psychodynamik der Sucht spezifische Modus die „Überlassung an ein unbelebtes Objekt“ ist, wobei diese „Überlassung“ selbst als Abwehr eines Abhängigkeitswunsches (durch Verschiebung auf das Suchtmittel) erscheint, dann kann dieser Modus vor dem Hintergrund der bisherigen Ausführungen im Grunde nicht auf einen einzigen (Abwehr-),Mechanismus“ reduziert werden (allein schon deshalb nicht, weil dieser Modus selbst das Ineinanderwirken von mindestens drei distinkten Abwehrmechanismen impliziert: die Verleugnung realer Abhängigkeit, die Projektion menschlicher Qualitäten auf das Suchtobjekt und schließlich die Verdrängung dieses Abwehrprozesses). Anstatt also den suchtspezifischen Modus isoliert als Resultat einer (scheinbar) einfachen „Verschiebung“ zu begreifen, wie Voigtel dies tut, erscheint es plausibler, ihn als Ausdruck einer komplexen „Abwehrorganisation“ [50] zu verstehen. Dieser Begriff erscheint geeignet, die Komplexität der suchtspezifischen Abwehr zu erfassen, insofern er mittlerweile konfliktdynamische und strukturelle Aspekte umfasst, etwa wenn zeitgenössische kleinianische Autoren [51] damit eine "fixierte Struktur" von Abwehrmechanismen i. S. einer strukturellen „Organisation“ [52] bezeichnen ${ }^{6}$. So beschreibt etwa E. ÓShaughnessy [53] die Abwehrorganisation als „pathologische Fixierung“, die, einmal gebildet, von nun an die „Beziehungen“ zwischen dem Subjekt und „seinen Objekten“ regelt. Mit Blick darauf erscheint die „Fixierung“ auf das Suchtmittel als organisierte Abwehr von Abhängigkeit und Bezo-

\footnotetext{
${ }^{6}$ Wobei es in diesem Zusammenhang erwähnenswert ist, dass gerade die kleinianischen Autoren (Joseph, Meltzer, Rosenfeld u. Steiner) immer wieder die enge Beziehung zwischen „pathologischer (Abwehr-)Organisation" und Sucht betont haben.
}

genheit, deren Kern der suchtspezifische Modus der „Überlassung“ ist. Dabei ist die „Organisation“ dieser Abwehr in dem Maße komplex, in dem ihr jeweils unterschiedliche, unbewusste Konfliktkonstellationen zugrunde liegen können und überdies auch unterschiedlichen „Ebenen“ bzw. „Niveaus“ der (strukturellen) Persönlichkeitsorganisation (neurotisch, borderline und psychotisch) entsprechen. Mit Blick darauf bestimmt dann die jeweils individuelle Kombination von Konflikt- und Strukturdimension Funktion und Wirkung des Suchtmittels wie auch die Ausprägung des Suchtgeschehens insgesamt. Die zentrale Aufgabe der psychodynamischen Suchtdiagnostik besteht demzufolge vor allem darin, diese psychologische(n) Funktion(en $)^{7}$ des Suchtmittels für den Süchtigen möglichst genau zu diagnostizieren: Welche unbewussten Konflikte werden durch dessen Einnahme „verarbeitet“, welche strukturellen Beeinträchtigungen bzw. Defizite dadurch kompensiert? Dazu liefert das Instrument der Operationalisierten Psychodynamischen Diagnostik (OPD) einen klinisch bedeutsamen Beitrag 8 .

Das gilt auch mit Blick auf die psychotherapeutische Praxis der Suchttherapie. Insofern es hier vorrangig um die Arbeit an der suchtspezifischen „Verschiebung“ des Abhängigkeitswunsches auf das Suchtmittel geht, erlaubt ein Instrument wie die OPD eine therapeutisch handhabbare und damit auch praktisch nützliche Differenzierung der süchtigen Abwehrorganisation. Wenn das primäre Ziel der Psychotherapie in der Arbeit an bzw. der Aufhebung der süchtigen Abwehr besteht, dann steht diese Zielvorstellung weder im Widerspruch noch in Konkurrenz zu aktuellen neurobiologischen bzw. pharmakologischen Erkenntnissen der Suchtforschung und -behandlung. So kann etwa die Einnahme des Medikaments Acamprosat das dranghafte Verlangen (craving), Alkohol zu trinken, durchaus dämpfen; was es nicht leisten kann, ist die Wirksamkeit des psychologischen Suchtmechanismus, d. h. die psychische Bereitschaft des Alkoholikers, sich dem Alkohol passiv zu überlassen, außer Kraft zu setzen. Dies bleibt ungeachtet aller möglichen begleitenden Medikation(en) eine nach wie vor vornehmlich psychotherapeutische Aufgabe.

\section{Literatur}

${ }^{1}$ Arbeitskreis OPD. Operationalisierte Psychodynamische Diagnostik. Grundlagen und Manual. Bern, Göttingen, Toronto, Seattle: Verlag Hans Huber 1998

2 Nitzgen D, Brünger M. Operationalisierte Psychodynamische Diagnostik in der Rehabilitationsklinik Birkenbuck. In: Schneider W, Freyberger $\mathrm{HJ}$ (Hrsg). Was leistet die OPD? Empirische Befunde und klinische Erfahrungen mit der Operationalisierten Psychodynamischen Diagnostik. Bern, Göttingen, Toronto, Seattle: Verlag Hans Huber 2000; $238-251$

${ }^{3}$ Nitzgen D. The Growing Terror Of No/thing. Überlegungen zur gruppenanalytischen Psychotherapie „süchtiger Objektbeziehungen“. In: Jahrbuch für Gruppenanalyse. Heidelberg: mattes Verlag 1995; Bd. 1: $77-93$

\footnotetext{
${ }^{7}$ Und eben nicht nur die neurobiologischen. Allein aus diesem Grund erscheint die von Tretter [54] unlängst beklagte „Substitution psychologischer Begriffe durch neurobiologische Begriffe“ (S. 73) in der suchttherapeutischen Praxis aus psychodynamischer Sicht grundsätzlich problematisch.

${ }^{8}$ Ich danke Herrn Prof. Dr. J. Küchenhoff für seine wohlwollend-kritische Durchsicht meiner Arbeit vor allem im Hinblick auf die OPD; seine Anmerkungen haben wesentlich zur Präzisierung des Textes beigetragen.
} 
${ }^{4}$ Lindner WV. Psychodynamik der Sucht. Persönlichkeitsstörungen. Theorie und Therapie 1998; 3: 125-134

${ }^{5}$ Rost WD. Psychoanalyse des Alkoholismus. Stuttgart: Klett Cotta 1987

${ }^{6}$ Kernberg OF. Borderline Störungen und pathologischer Narzißmus. Frankfurt: Suhrkamp 1979

${ }^{7}$ Wurmser L. Die verborgene Dimension. Psychodynamik des Drogenzwangs. Göttingen: Vandenhoeck\&Ruprecht 1997

${ }^{8}$ Wanke K, Bühringer G. Grundstörungen der Sucht. Berlin: Springer 1991

${ }^{9}$ Krause R. Allgemeine Psychoanalytische Krankheitslehre. Grundlagen. Stuttgart, Berlin, Köln: Kohlhammer 1997; Bd. I

${ }^{10}$ Krause R. Allgemeine Psychoanalytische Krankheitslehre. Grundlagen. Stuttgart, Berlin, Köln: Kohlhammer 1997; Bd. I

${ }^{11}$ Hopper EA. Psychoanalytic theory of drug addiction. The International Journal of Psychoanalysis 1995; 76: 1121 - 1142

12 Heigel-Evers A, Schultze-Dierbach E, Standke D. Grundstörungen bei Abhängigkeit und Sucht aus tiefenpsychologischer Sicht. In: Wanke $\mathrm{K}$, Bühringer G (Hrsg). Grundstörungen der Sucht. Berlin, Heidelberg, New York: Springer 1991; 37-55

${ }^{13}$ Sachs H. Zur Genese der Perversionen. Psyche 1971; 25: 287-297

${ }^{14}$ Freud A. Wege und Irrwege in der Kinderentwicklung. Stuttgart: Ernst Klett Verlag 1968

15 Voigtel R. Die Überlassung an das unbelebte Objekt. Zur begrifflich-diagnostischen Abgrenzung der Sucht. Psyche 1996; 8: 715 - 742

${ }^{16}$ von Gebsattel VE. Zur Psychopathologie der Sucht. In: Prolegomena einer medizinischen Anthropologie. Berlin, Göttingen, Heidelberg: Springer Verlag 1954

17 Voigtel R. Die Überlassung an das unbelebte Objekt. Zur begrifflich-diagnostischen Abgrenzung der Sucht. Psyche 1996; 8: 715-742

${ }^{18}$ Simmel E. Alkoholismus und Sucht. In: Herrmanns VL, Schulz-Venrath U (Hrsg) (Hrsg). Psychoanalyse und ihre Anwendungen. Ausgewählte Schriften. Frankfurt a.M: Fischer TB 1993; 289-313

19 Voigtel R. Die Überlassung an das unbelebte Objekt. Zur begrifflich-diagnostischen Abgrenzung der Sucht. Psyche 1996; 8: 715 - 742

${ }^{20}$ Geismar-Wieviorka S. Les Toxicomanes. Paris: Presses Universitaire 1995

${ }^{21}$ Voigtel R. Die Überlassung an das unbelebte Objekt. Zur begrifflich-diagnostischen Abgrenzung der Sucht. Psyche 1996; 8: 715 - 742

${ }^{22}$ Heigel-Evers A, Schultze-Dierbach E, Standke D. Grundstörungen bei Abhängigkeit und Sucht aus tiefenpsychologischer Sicht. In: Wanke K, Bühriinger G (Hrsg). Grundstörungen der Sucht. Berlin, Heidelberg, New York: Springer 1991; 37-55

${ }^{23}$ Heigel-Evers A, Standke G, Wienen G. Sozialisationsstörungen und Sucht - psychoanalytische Aspekte. In: Feuerlein W (Hrsg). Sozialisationsstörungen und Sucht - Entstehungsbedingungen, Folgen, therapeutische Konsequenzen. Wiesbaden: Akademische Verlagsgesellschaft 1981

${ }^{24}$ Kernberg OF. Die Bedeutung neuerer psychoanalytischer und psychodynamischer Konzepte für die Befunderhebung und Klassifikation von Persönlichkeitsstörungen. In: Schauenburg H, Freyberger HJ, Cierpka $\mathrm{M}$ et al (Hrsg). OPD in der Praxis. Konzepte, Anwendungen, Ergebnisse der Operationalisierten Psychodynamischen Diagnostik. Göttingen: Verlag Hans Huber 1996; 55-68

${ }^{25}$ Voigtel R. Die Überlassung an das unbelebte Objekt. Zur begrifflich-diagnostischen Abgrenzung der Sucht. Psyche 1996; 8

${ }^{26}$ Voigtel R. Die Überlassung an das unbelebte Objekt. Zur begrifflich-diagnostischen Abgrenzung der Sucht. Psyche 1996; 8

${ }^{27}$ Sachs H. Zur Genese der Perversionen. Psyche 1971; 25: 287-297

28 Voigtel R. Die Überlassung an das unbelebte Objekt. Zur begrifflich-diagnostischen Abgrenzung der Sucht. Psyche 1996; 8: 715 - 742

${ }^{29}$ Cooper AM. The unconscious core of perversion. In: Fogel GI, Myers WA (Hrsg). Perversions and near-perversions in clinical practice. New psychoanalytic perspectives. New York: Yale University Press 1991

${ }^{30}$ Voigtel R. Die Überlassung an das unbelebte Objekt. Zur begrifflich-diagnostischen Abgrenzung der Sucht. Psyche 1996; 8: 715 - 742

${ }^{31}$ McDougall J. Theater der Seele. Illusion und Wahrheit auf der Bühne der Psychoanalyse. München, Wien: Verlag Internationale Psychoanalyse 1988

${ }^{32}$ Winnicott DW. Übergangsobjekte und Übergangsphänomene. In: Winnicott DW (Hrsg). Von der Kinderheilkunde zur Psychoanalyse. Frankfurt a.M.: Fischer TB 1983; 300-318

${ }^{33}$ McDougall J. Die Couch ist kein Prokrustesbett. Zur Psychoanalyse der menschlichen Sexualität. Stuttgart: Verlag Internationale Psychoanalyse 1997
${ }^{34}$ Khantzian E. The Self-Medication Hypothesis of Addictive Disorders: Focus on Heroine and Cocaine Dependence. The American Journal of Psychiatry 1985; 142 (11): $1259-1264$

35 Voigtel R. Die Überlassung an das unbelebte Objekt. Zur begrifflich-diagnostischen Abgrenzung der Sucht. Psyche 1996; 8: 715-742

${ }^{36}$ Voigtel R. Die Überlassung an das unbelebte Objekt. Zur begrifflich-diagnostischen Abgrenzung der Sucht. Psyche 1996; 8: 715-742

${ }^{37}$ Lindner WV. Psychodynamik der Sucht. Persönlichkeitsstörungen. Theorie und Therapie 1998; 3: 125-134

38 Voigtel R. Die Überlassung an das unbelebte Objekt. Zur begrifflich-diagnostischen Abgrenzung der Sucht. Psyche 1996; 8: 715-742

${ }^{39}$ Arbeitskreis OPD. Operationalisierte Psychodynamische Diagnostik. Grundlagen und Manual. Bern, Göttingen, Toronto, Seattle: Verlag Hans Huber 1998

${ }^{40}$ Arbeitskreis OPD. Operationalisierte Psychodynamische Diagnostik. Grundlagen und Manual. Bern, Göttingen, Toronto, Seattle: Verlag Hans Huber 1998

${ }^{41}$ Nitzgen D, Brünger M. Operationalisierte Psychodynamische Diagnostik in der Rehabilitationsklinik Birkenbuck. In: Schneider W, Freyberger $\mathrm{HJ}$ (Hrsg). Was leistet die OPD? Empirische Befunde und klinische Erfahrungen mit der Operationalisierten Psychodynamischen Diagnostik. Bern, Göttingen, Toronto, Seattle: Verlag Hans Huber 2000; 238 - 251

42 Arbeitskreis OPD. Operationalisierte Psychodynamische Diagnostik. Grundlagen und Manual. Bern Göttingen Toronto Seattle: Verlag Hans Huber 1998

${ }^{43}$ Kernberg OF. Die Bedeutung neuerer psychoanalytischer und psychodynamischer Konzepte für die Befunderhebung und Klassifikation von Persönlichkeitsstörungen. In: Schauenburg H, Freyberger HJ, Cierpka $\mathrm{M}$ et al (Hrsg). OPD in der Praxis. Konzepte, Anwendungen, Ergebnisse der Operationalisierten Psychodynamischen Diagnostik. Göttingen: Verlag Hans Huber 1996; 55-68

${ }^{44}$ Reymann G. Evidenzbasierte Psychotherapie. Der Beitrag psychodynamischer Therapieverfahren. SUCHT 2002; 3: 182 - 193

${ }^{45}$ Reymann G, Zbikowski A, Martin K et al. Erfahrungen mit der Anwendung von Operationalisierter Psychodynamischer Diagnostik bei Alkoholkranken. In: Schneider W, Freyberger HJ (Hrsg). Was leistet die OPD? Empirische Befunde und klinische Erfahrungen mit der Operationalisierten Psychodynamischen Diagnostik. Bern, Göttingen, Toronto, Seattle: Verlag Hans Huber 2002; 229 - 237

${ }^{46}$ Nitzgen D, Brünger M. Operationalisierte Psychodynamische Diagnostik in der Rehabilitationsklinik Birkenbuck. In: Schneider W, Freyberger $\mathrm{HJ}$ (Hrsg). Was leistet die OPD? Empirische Befunde und klinische Erfahrungen mit der Operationalisierten Psychodynamischen Diagnostik. Bern, Göttingen, Toronto, Seattle: Verlag Hans Huber 2000; 238 - 251

${ }^{47}$ Grande T, Oberbracht C, Rudolf G. Einige empirische Zusammenhänge zwischen den Achsen „Beziehung“, „Konflikt“ und „Struktur“. In: Schauenburg H, Freyberger, HJ, Cirpka M et al (Hrsg). OPD in der Praxis. Konzepte, Anwendungen, Ergebnisse der Operationalisierten Psychodynamischen Diagnostik. Bern, Göttingen, Toronto, Seattle: Verlag Hans Huber 1998; 121 - 139

${ }^{48}$ Nitzgen D, Brünger M. Operationalisierte Psychodynamische Diagnostik in der Rehabilitationsklinik Birkenbuck. In: Schneider W, Freyberger $\mathrm{HJ}$ (Hrsg). Was leistet die OPD? Empirische Befunde und klinische Erfahrungen mit der Operationalisierten Psychodynamischen Diagnostik. Bern, Göttingen, Toronto, Seattle: Verlag Hans Huber 2000; 238 - 251

${ }^{49}$ Kunzke D, Strauß B, Burtscheidt W. Zur Wirksamkeit der psychoanalytisch orientierten Gruppenpsychotherapie des Alkoholismus - Literaturübersicht. Gruppenpsychotherapie \& Gruppendynamik 2002; 1 : $53-70$

${ }^{50}$ Hoffer W. Defensive process and defensive organisation: their place in psycho-analytic technique. Int J o Psychoanal 1954; 35 (2): 194-198

51 O'Shaughnessy E. Klinische Untersuchung einer Abwehrorganisation. In: Bott-Spillius E (Hrsg). Melanie Klein Heute. Entwicklungen in Theorie und Praxis, Beiträge zur Theorie. München: Verlag Internationale Psychoanalyse 1990; Bd. 1: 367-390

52 Hinshelwood RD. Wörterbuch der kleinianischen Psychoanalyse. Stuttgart: Verlag Internationale Psychoanalyse 1993

53 ÓShaughnessy E. Klinische Untersuchung einer Abwehrorganisation. In: Bott-Spillius E (Hrsg). Melanie Klein Heute. Entwicklungen in Theorie und Praxis, Beiträge zur Theorie. München: Verlag Internationale Psychoanalyse 1990; Bd. 1: 367-390

${ }^{54}$ Tretter F. Wie praktisch ist die „Suchttheorie“? Wissenschaftstheoretische Probleme der Suchtforschung und Suchthilfe. Sucht aktuell 2000; $1: 69-74$ 